


\section{The Gaussian-BGK model of Boltzmann equation with small prandtl number}

Pierre Andries - Patrick Le Tallec - Jean-Philippe Perlat - Benoit Perthame

\section{No 3716}

June 24, 1999

THÈME 4

\section{apport}

derecherche 



\title{
The Gaussian-BGK model of Boltzmann equation with small prandtl number
}

\author{
Pierre Andries* - Patrick Le Tallec ${ }^{\dagger}$ - Jean-Philippe Perlat ${ }^{\ddagger}$ - \\ Benoit Perthame
}

Thème 4 - Simulation et optimisation

de systèmes complexes

Projet M3N

Rapport de recherche $\mathrm{n}^{\circ} 3716$ - June 24,1999 - 24 pages

\begin{abstract}
In this paper we prove the entropy inequality for the GaussianBGK model of Boltzmann equation. This model, also called ellipsoidal statistical model, was introduced in order to fit realistic values of the transport coefficients (Prandtl number, second viscosity) in the Navier-Stokes approximation, which cannot be achieved by the usual relaxation towards isotropic Maxwellians introduced in standard BGK models.

Moreover, we introduce new entropic kinetic models for polyatomic gases which suppress the internal energy variable in the phase space by using two distribution functions (one for particles mass and one for their internal energy). This reduces the cost of their numerical solution while keeping a kinetic description well adapted to desequilibrium regions.
\end{abstract}

Key-words: Kinetic Theory, BGK model, Entropy inequality, ChapmanEnskog expansion

(Résumé : tsvp)

*M3N,email: pierre.andries@inria.fr

$\dagger$ CEREMADE,Université Paris-Dauphine, 75775-Paris cedex 16, email: letallec@ceremade.dauphine.fr

‡ M3N,email: jean-philippe.perlat@inria.fr

$\S$ M3N,email: benoit.perthame@ens.fr 


\section{Le modèle BGK gaussien de l'équation de Boltzmann avec faible nombre de Prandtl}

Résumé : Dans ce travail nous prouvons l'inégalité d'entropie pour le modèle BGK Gaussien de l'équation de Boltzmann. Ce modèle, appelé aussi statistique-éllipsoidal, fut introduit dans le but d'obtenir des coéfficients de transport (nombre de Prandtl, viscosité seconde) réalistes à la limite fluide, ce qui ne peut être obtenu par la relaxation vers une maxwellienne anisotrope introduite dans le modèle de BGK standard.

De plus, nous introduisons de nouveaux modèles cinétiques pour les gaz poliatomiques qui suppriment la variable d'énergie interne dans l'espace des phases par l'utilisation de deux fonctions de distribution (une pour la masse des particules et une pour leur énergie interne). Cela réduit le cout de la résolution numérique tout en gardant une description cinétique bine adaptée aux régions de déséquilibre.

Mots-clé : Théorie cinétique, Modèle de BGK, Inégalité d'entropie, Développement de Chapman-Enskog 


\section{Contents}

1 Introduction 4

2 Gaussian BGK equation: the monoatomic case 6

2.1 Some notations . . . . . . . . . . . . . . 6

2.2 Gaussian model . . . . . . . . . . . . . . . . . 7

2.3 entropy inequality . . . . . . . . . . . . . 8

3 Gaussian model : the polyatomic case 11

3.1 The Gaussian model ................. . 11

3.2 Entropy inequality ................. 14

4 Transport coefficients $\quad 18$

5 Polyatomic case. Description with two distribution functions 20

6 Appendix. Brunn-Minkowsky inequality. 22 


\section{Introduction}

At high altitudes or in rarefied regimes, a gas is best modeled at a microscopic scale, as a collection of particles characterized by their velocity $\underline{v}$ and position $\underline{x}$. The relevant mathematical model is then the Boltzmann equation,

$$
\partial_{t} f+\underline{v} \cdot \nabla_{\underline{x}} f=Q(f) .
$$

which governs the evolution of the density of particles $f$ in the phase i.e. in the monoatomic case $f=f(t, \underline{x}, \underline{v}), t \geq 0,(\underline{x}, \underline{v}) \in I R^{3} \times I R^{3}$, and in the polyatomic case $f=f(t, \underline{x}, \underline{v}, I)$, where the additional parameter $I \geq 0$ is an internal energy parameter. This equation is closely related to the NavierStokes system which governs the evolution of macroscopic density, momentum and energy

$$
\partial_{t}\left(\begin{array}{l}
\rho \\
\rho u_{i} \\
E
\end{array}\right)+\partial_{x_{j}} \cdot\left(\begin{array}{l}
\rho u_{i} \\
\rho u_{i} u_{j}+P \delta_{i j} \\
E u_{j}+P u_{j}
\end{array}\right)=\partial_{x_{j}} \cdot\left(\begin{array}{l}
0 \\
\sigma_{i j} \\
u_{i} \sigma_{i j}+\kappa \partial_{x_{j}} T
\end{array}\right),
$$

where the pressure is given by the polytropic law

$$
P=\rho R T=(\gamma-1) \rho e,
$$

the viscosity tensor is given by

$$
\sigma_{i j}=\mu\left(\partial_{x_{j}} u_{i}+\partial_{x_{i}} u_{j}-\alpha \delta_{i j} \operatorname{div} u\right)
$$

the total energy by $E=\frac{1}{2} \rho|u|^{2}+\rho e$. Finally, the viscosity $\mu(T)$, the second viscosity $\alpha \mu$ and heat conductivity $\kappa(T)$ are given coefficients. Here $R$ denotes the ordinary gas constant per unit mass.

Since the quadratic collision operator $Q(f)$ has a rather complex form, simpler models have been introduced and are commonly used (see Loyalka and als [14], Aoki and als [2] for example). These models should respect the basic relaxation properties of the gas under study and be reliable when computing boundary layers or shock waves in transitional flows (that is in regimes where the gas is dense but not completely in thermodynamic equilibrium). On the other hand, they should be easier to handle numerically, and should simplify the study of transitional regimes. More precisely, we are looking for models whose hydrodynamic limits can be easily accessed by Chapman-Enskog or by asymptotic moment expansions and have the right transport coefficients. The simplest model is the so-called BGK model introduced by Bhatnagar, Gross and Krook [3]. It is based on relaxation towards local Maxwellians

$$
Q(f)=\frac{1}{\varepsilon}(\mathcal{M}[f]-f)
$$


This model has the advantage of describing the right fluid limit. But in the Chapman-Enskog expansion, the transport coefficients, that is $\mu, \alpha$, and $\kappa$ obtained at the Navier-Stokes level are not satisfactory. In particular, the Prandtl number defined by

$$
\operatorname{Pr}=\frac{\gamma}{\gamma-1} \frac{R \mu}{\kappa}
$$

and relating the viscosity to the heat conductivity is equal to 1 . For most gases, we have $\operatorname{Pr}<1$. In particular, the hard-sphere model for a monoatomic gas $(\gamma=5 / 3)$ in Boltzmann equation leads to a Prandtl number very close to $2 / 3$. Many variants of the BGK model have been proposed in order to give the correct Navier-Stokes heat conduction (see Brun [6], Cercignani [8]) but none of them satisfies simultaneously the three basic requirements of giving nonnegative distributions, of predicting a Prandtl number less than 1 , and of verifying the fundamental $\mathrm{H}$-Theorem

$$
\int Q(f) \ln f d v \leq 0
$$

which implies the classical and fundamental entropy inequality

$$
\begin{gathered}
\partial_{t} \int H(f) d \underline{v}+\operatorname{div} \int \underline{v} H(f) d \underline{v} \leq 0, \\
H(f)=f \ln f .
\end{gathered}
$$

Actually, the model proposed by Bouchut and Perthame [5] can do this, but it is too complex for numerical purposes. In Levermore [12] a BGK system is proposed but it can only reach Prandtl numbers larger than 1 for nonnegative distributions. A model was proposed by Holway [9] which gives non negative distribution and a Prandtl number less than one, but to prove the entropy inequality for this model has been a long standing open problem. Nevertheless various studies and numerical simulations have been conducted with results in good agreement with experimental data, for references see [8] and in particular $[7]$.

The actual form of the model, called ellipsoidal statistical or Gaussian model, involves non-convex quantities for $\frac{2}{3} \leq \operatorname{Pr} \leq 1$. This fact made unlikely that the entropy inequality (a convex relation) might hold true and the problem of proving or disproving the H-Theorem was left open.

In this paper, we solve this long standing question by showing that the model satisfies indeed the entropy inequality for the range $\frac{2}{3} \leq \operatorname{Pr} \leq 1$. A second purpose of this paper is to extend this model and the associated ChapmanEnskog expansion to polyatomic gases. Two kinetic descriptions are in fact possible here. The classical description uses a distribution function $f(t, \underline{v}, \underline{x}, I)$ which also contains an internal energy parameter $I$ at the kinetic level (see

RR $n^{\circ} 3716$ 
Lifschitz-Pitaevski [13] for a general setting, or Morse [16] for the precise BGK model). The other description to be detailed in this paper is to use two density functions, one for mass, and one for internal energy. It was used for Euler equations by Khobalatte and Perthame [11] and has the advantage of reducing the number of independent variables which is very useful for numerical purposes (see Mieussens [15] for instance). In a forthcoming paper, we will present comparisons between numerical tests using Boltzmann's quadratic kernel and the ellipsoidal model (Andries and al. [1]).

The outline of this paper is as follows. In section 2, we describe the Gaussian model for a monoatomic gas, and we prove the entropy property (1.6) for this model. In Section 3, we extend the model and the entropy property to the polyatomic case using an additional internal degree of freedom. The Chapman-Enskog expansion is performed in Section 4, and we show that the range of Prandtl numbers $2 / 3 \leq \operatorname{Pr}<\infty$ can indeed be reached while leaving free the second viscosity coefficient $\alpha$ in the polyatomic case (in the monoatomic case we can only reach the classical Stokes relation $\alpha=2 / 3$ as for the binary Boltzmann collisional operator). Finally, the description with two distribution functions is treated in section 5 .

\section{Gaussian BGK equation: the monoatomic case}

In this Section we consider a monoatomic gas i.e. $\gamma=5 / 3$ in 1.3 . We recall the Gaussian model which allows to recover the range of Prandtl numbers $\frac{2}{3} \leq \operatorname{Pr} \leq 1$, and we show that it satisfies the entropy properties $(1.6),(1.7)$.

\subsection{Some notations}

Boltzmann's collision operator $Q(f)(\underline{x}, \underline{v}, t)$, which describes the variation of the distribution function due to binary collisions between particles, conserves mass, velocity and energy

$$
\int_{\underline{v} \in R^{3}} Q(f)(\underline{x}, \underline{v}, t) \phi(\underline{v}) d \underline{v}=0
$$

for $\phi(\underline{v})=\left\{1, \underline{v}, \frac{1}{2}|v|^{2}\right\}$, and satisfies the local entropy dissipation inequality (1.6). These properties are true for the simpler B.G.K. operator (1.4) which describes the relaxation of $f$ to the local Maxwellian equilibrium

$$
\mathcal{M}[f]=\frac{\rho}{(2 \pi R T)^{3 / 2}} \exp \left(-\frac{|v-u|^{2}}{2 R T}\right) .
$$

It is defined by using the macroscopic density $\rho$, velocity $\underline{u}$ and translational temperature $T$ of the original non-negative distribution $f$ obtained through 
the moments

$$
\begin{gathered}
\rho(\underline{x}, t)=<f>, \\
\underline{u}(\underline{x}, t)=\frac{1}{\rho}<\underline{v} f>, \\
T=\frac{1}{3 R \rho(\underline{x}, t)}<|\underline{v}-\underline{u}(\underline{x}, t)|^{2} f>,
\end{gathered}
$$

with the notation

$$
<f>(\underline{x}, t)=\int_{\underline{v} \in R^{3}} f(\underline{x}, t, \underline{v}) d \underline{v} .
$$

In order to introduce the Gaussian model, we need further notations. We define

$$
\begin{gathered}
P=\rho R T \quad \text { (kinetic pressure), } \\
E=\frac{1}{2}<|v|^{2} f>=\frac{1}{2} \rho\left(|u|^{2}+3 R T\right) \quad \text { (total energy), }
\end{gathered}
$$

and the opposite of the stress tensor

$$
\underline{\underline{\Theta}}=\frac{1}{\rho}<\underline{c} \otimes \underline{c} f>
$$

with $\underline{c}=\underline{v}-\underline{u}(\underline{x}, t)$ denoting the relative velocity. Therefore the translational temperature is related to the stress tensor by

$$
T=\frac{1}{3 R} \operatorname{tr} \underline{\underline{\Theta}}
$$

We finally introduce the corrected tensor

$$
\underline{\underline{\mathcal{T}}}=(1-\nu) R T \underline{\underline{I d}}+\nu \underline{\underline{\Theta}}
$$

which can be viewed as a linear combination of the initial stress tensor $\underline{\underline{\Theta}}$ and of the isotropic stress tensor $R T \underline{\underline{I d}}$ developed by a Maxwellian distribution.

\subsection{Gaussian model}

The Gaussian model introduces a corrected BGK collision operator by replacing the local equilibrium Maxwellian by the Gaussian $\mathcal{G}[f]$ defined by

$$
\mathcal{G}[f]=\frac{\rho}{\sqrt{\operatorname{det}(2 \pi \underline{\mathcal{T}})}} \exp \left(-\frac{1}{2}(\underline{v}-\underline{u}) \cdot \underline{\underline{\mathcal{T}}}^{-1} \cdot(\underline{v}-\underline{u})\right) .
$$

The corresponding collision operator is now

$$
Q(f)=\frac{P}{\mu(1-\nu)}(\mathcal{G}[f]-f),
$$

RR $n^{\circ} 3716$ 
where the parameter $-\frac{1}{2} \leq \nu<1$ is used to modify the value of the Prandtl number through the formula (to be proved later)

$$
\frac{2}{3} \leq \operatorname{Pr}=\frac{5}{2} \frac{R \mu}{\kappa}=\frac{1}{1-\nu} \leq+\infty .
$$

It first follows from the above definitions, using for example the change of variables $\underline{\tilde{c}}=\underline{\underline{T}}^{-1 / 2} \cdot(\underline{v}-\underline{u})$, that

$$
\begin{aligned}
<f>=\rho & =<\mathcal{G}[f]> \\
<\underline{v} f>=\rho u & =<\underline{v} \mathcal{G}[f]> \\
<\frac{|v|^{2}}{2} f>=E & =<\frac{|v|^{2}}{2} \mathcal{G}[f]>
\end{aligned}
$$

and

$$
\begin{gathered}
<(\underline{v}-\underline{u}) \otimes(\underline{v}-\underline{u}) f>=\rho \underline{\underline{\Theta}}, \\
<(\underline{v}-\underline{u}) \otimes(\underline{v}-\underline{u}) \mathcal{G}[f]>=\rho \underline{\underline{\mathcal{T}}} .
\end{gathered}
$$

In particular, this means that the collision operator (2.10) does indeed conserve mass, momentum and energy as imposed.

\section{3 entropy inequality}

To prove the entropy inequality (1.6) (H-Theorem) we introduce the entropy constrained minimization problem

$$
S(\rho, \underline{u}, \underline{\underline{\mathcal{T}}})=\min _{g \in \mathcal{X}}<H(g)>,
$$

where $\mathcal{X}=\mathcal{X}(\rho, \underline{u}, \underline{\underline{\mathcal{T}}})$ is the set defined by

$$
\begin{aligned}
\mathcal{X} & =\left\{g \geq 0,\left(1+|v|^{2}\right) g \in L^{1}\left(I R^{3}\right),\right. \\
& <g>=\rho,<\underline{v} g>=\rho \underline{u},<\underline{v} \otimes \underline{v} g>=\rho \underline{u} \otimes \underline{u}+\rho \underline{\mathcal{T}}\} .
\end{aligned}
$$

We have the following proposition

\section{Proposition 2.1.}

For symmetric positive definite tensor $\underline{\underline{\Theta}}$ and $-1 / 2 \leq \nu<1$ we have

(i) the tensor $\underline{\underline{\mathcal{T}}}$ defined in (2.8) is symmetric positive definite and the set $\mathcal{X}(\rho, \underline{u}, \underline{\underline{\mathcal{T}}})$ is not empty,

(ii) the unique minimizer in (2.13) is the Gaussian $\mathcal{G}[f]$ defined in (2.9),

(iii) the entropy of the Gaussian $\mathcal{G}[f]$ satisfies

$$
<H(\mathcal{G})>=S(\rho, \underline{u}, \underline{\underline{\mathcal{T}}}) \leq S(\rho, \underline{u}, \underline{\underline{\Theta}}) \leq<H(f)>
$$

(iv) consequently the entropy inequalities (1.6), (1.7) hold,

(v) the equality $\langle H(\mathcal{G})\rangle=<H(f)>$ implies $f=\mathcal{M}$. 
proof.The first and second point will be proved later on in the more general polyatomic case, thus we refer to section 3 for a proof. For the third point, using (ii) we first compute

$$
\begin{aligned}
S(\rho, \underline{u}, \underline{\mathcal{T}}) & =\ln \left(\frac{\rho}{\sqrt{\operatorname{det}(2 \pi \underline{\mathcal{T}})}}\right)<\mathcal{G}>-\frac{1}{2}<\underline{\mathcal{T}}^{-1}:(\underline{c} \otimes \underline{c}) \mathcal{G}>-<\mathcal{G}> \\
& =\rho \ln \left(\frac{\rho}{\sqrt{\operatorname{det}(2 \pi \underline{\underline{T}})}}\right)-\frac{5}{2} \rho,
\end{aligned}
$$

yielding

$$
\begin{aligned}
S(\rho, \underline{u}, \underline{\underline{\mathcal{T}}})-S(\rho, \underline{\underline{u}}, \underline{\underline{\Theta}}) & =\rho \ln \left(\frac{\rho}{\sqrt{\operatorname{det}(2 \pi \underline{\mathcal{T}})}}\right)-\rho \ln \left(\frac{\rho}{\sqrt{\operatorname{det}(2 \pi \underline{\underline{\Theta}})}}\right) \\
& =\frac{1}{2} \rho \ln \frac{\operatorname{det} \underline{\underline{\underline{\Theta}}}}{\operatorname{det} \underline{\underline{\mathcal{T}}}}
\end{aligned}
$$

Thus, we just have to prove that

$$
\operatorname{det} \underline{\underline{\mathcal{T}}} \geq \operatorname{det} \underline{\underline{\Theta}}
$$

This is mainly a consequence of the Brunn-Minkowsky inequality

$$
\operatorname{det}(a A+(1-a) B)>(\operatorname{det} A)^{a}(\operatorname{det} B)^{1-a}
$$

for $0<a<1$ and $A, B$ positive symmetric matrices, $A \neq B$. This inequality can be evidently extended to a general convex combination of matrices. This inequality will be proved later on in the appendix. Here we cannot apply this result directly to

$$
\underline{\underline{\mathcal{T}}}=\nu \underline{\underline{\Theta}}+(1-\nu) R T \underline{\underline{I d}}
$$

because $\nu$ may be negative. Instead, we introduce, in a diagonal basis for $\underline{\underline{\Theta}}$ the matrices

$$
\Lambda_{1}=\left(\begin{array}{ccc}
\lambda_{1} & 0 & 0 \\
0 & \lambda_{2} & 0 \\
0 & 0 & \lambda_{3}
\end{array}\right), \Lambda_{2}=\left(\begin{array}{ccc}
\lambda_{2} & 0 & 0 \\
0 & \lambda_{3} & 0 \\
0 & 0 & \lambda_{1}
\end{array}\right), \Lambda_{3}=\left(\begin{array}{ccc}
\lambda_{3} & 0 & 0 \\
0 & \lambda_{1} & 0 \\
0 & 0 & \lambda_{2}
\end{array}\right)
$$

where $\lambda_{i}$ are the eigenvalues of $\underline{\underline{\Theta}}$. Then, $\underline{\underline{\mathcal{T}}}$ is also diagonal and since $R T=$ $\left(\lambda_{1}+\lambda_{2}+\lambda_{3}\right) / 3$, it is easy to obtain from the definition of $\underline{\underline{\mathcal{T}}}$

$$
\underline{\underline{\mathcal{T}}}=\frac{1+2 \nu}{3} \Lambda_{1}+\frac{1-\nu}{3} \Lambda_{2}+\frac{1-\nu}{3} \Lambda_{3}
$$

RR $n^{\circ} 3716$ 
For $-\frac{1}{2} \leq \nu \leq 1$ this is now a convex combination of three matrices and we may apply (2.16). After noticing that

$$
\operatorname{det} \Lambda_{1}=\operatorname{det} \Lambda_{2}=\operatorname{det} \Lambda_{3}=\lambda_{1} \lambda_{2} \lambda_{3}=\operatorname{det} \underline{\underline{\Theta}},
$$

we obtain

$$
\operatorname{det} \underline{\underline{\mathcal{T}}} \geq \operatorname{det} \underline{\underline{\Theta}}
$$

Therefore, the inequality (2.15) holds true for $1-\nu \geq 0$, and $1+2 \nu \geq 0$, and the proof of (iii) is complete.

We deduce (iv) as a consequence of (iii) and of the convexity relation

$$
H^{\prime}(f)(\mathcal{G}-f) \leq H(\mathcal{G})-H(f) .
$$

Then, the BGK model (2.10) satisfies the H-Theorem for all $\nu \in[-1 / 2,1]$ because, thanks to the convexity of $H$ we have

$$
\begin{aligned}
\partial_{t} \int_{I R^{3}} H(f) d \underline{v}+\nabla_{\underline{x}} \cdot \int_{I R^{3}} \underline{v} H(f) d \underline{v} & =\frac{\rho R T}{\mu(1-\nu)} \int_{I R^{3}} H^{\prime}(f)(\mathcal{G}[f]-f) d \underline{v} \\
& \leq \frac{\rho R T}{\mu(1-\nu)} \int_{I R^{3}}(H(\mathcal{G}[f])-H(f)) d \underline{v} \\
& \leq 0 .
\end{aligned}
$$

To prove $(\mathrm{v})$, we assume $\langle H(\mathcal{G})>=<H(f)>$. Then the point (iii) gives

$$
<H(\mathcal{G})>=S(\rho, \underline{u}, \underline{\underline{\mathcal{T}}})=S(\rho, \underline{u}, \underline{\underline{\Theta}})=<H(f)>.
$$

But, using (ii) the equality $S(\rho, \underline{u}, \underline{\underline{\Theta}})=<H(f)>$ implies by point (ii) that $f$ is the gaussian

$$
f=\mathcal{G}_{\underline{\Theta}}[f]=\frac{\rho}{\sqrt{\operatorname{det}(2 \pi \underline{\underline{\Theta}})}} \exp \left(-\frac{1}{2}(\underline{v}-\underline{u}) \cdot \underline{\underline{\Theta}}^{-1} \cdot(\underline{v}-\underline{u})\right) .
$$

Next, the equality $(2.14)$ also gives $S(\rho, \underline{u}, \underline{\mathcal{T}})=S(\rho, \underline{u}, \underline{\underline{\Theta}})$. These quantities can be computed exactly following the lines of point (iii), and (2.15) becomes

$$
\operatorname{det} \underline{\underline{\mathcal{T}}}=\operatorname{det} \underline{\underline{\Theta}} \text {. }
$$

With the notations (2.17), this gives

$$
\operatorname{det}\left(\frac{1+2 \nu}{3} \Lambda_{1}+\frac{1-\nu}{3} \Lambda_{2}+\frac{1-\nu}{3} \Lambda_{3}\right)=\operatorname{det} \underline{\underline{\Theta}} \text {. }
$$

This is the equality case of the strictly convex inequality (2.16) and since $\nu<1$, this equality implies

$$
\Lambda_{2}=\Lambda_{3},
$$

from wich we deduce immediatly

$$
\lambda_{1}=\lambda_{2}=\lambda_{3}=R T, \underline{\underline{\Theta}}=\underline{\underline{I d}} .
$$

Then, we use the gaussian structure of $f,(2.19)$, and (2.20) to obtain $f=\mathcal{M}$. 


\section{Gaussian model : the polyatomic case}

The extension to polyatomic gases can be performed using an internal energy parameter $I$ which takes into account the (continuous) degrees of freedom in a general way. This kind of formalism has been widely used, see [6], [11], [13].

\subsection{The Gaussian model}

The polyatomic distribution function $f(t, \underline{x}, \underline{v}, I)$ describes the number of particles with position $\underline{x}$, velocity $\underline{v}$ and internal energy $\epsilon(I)=I^{2 / \delta}$ at time $t$, and defines the macroscopic quantities $(2.2),(2.3),(2.6)$ by

$$
\begin{gathered}
\rho(\underline{x}, t)=<<f>>, \\
\underline{u}(\underline{x}, t)=\frac{1}{\rho}<<\underline{v} f>>, \\
E(\underline{x}, t)=<<\left(\frac{1}{2}|v|^{2}+I^{2 / \delta}\right) f>>=\frac{1}{2} \rho|u|^{2}+\rho e,
\end{gathered}
$$

under the new notation

$$
<<f>>(\underline{x}, t)=\int_{\underline{v} \in R^{3}, I \in R^{+}} f(t, \underline{x}, \underline{v}, I) d \underline{v} d I .
$$

Here, the ratio of specific heats $\gamma=\frac{C_{p}}{C_{v}}$ and the number of additional degrees of freedom of the gas $\delta$ are related by

$$
\gamma=\frac{\delta+5}{\delta+3}
$$

By example, for a diatomic gas, we have $\delta=2$ and thus $\gamma=1.4$.

The specific internal energy $e=\frac{1}{\rho} E-\frac{1}{2}|u|^{2}$ can be divided here in two parts, the internal energy of translational motion $e_{t r}$ and the energy associated with the internal structure $e_{\text {int }}$ :

$$
\begin{gathered}
e=e_{t r}+e_{i n t}, \\
e_{t r}=\frac{1}{\rho}<<\frac{1}{2}|v-u|^{2} f>>, \\
e_{i n t}=\frac{1}{\rho}<<I^{2 / \delta} f>>.
\end{gathered}
$$

We associate to these energies the corresponding temperatures $T_{e q}, T_{t r}, T_{\text {int }}$

RR $n^{\circ} 3716$ 


$$
\begin{gathered}
e=\frac{3+\delta}{2} R T_{e q}, \\
e_{t r}=\frac{3}{2} R T_{t r}, \\
e_{i n t}=\frac{\delta}{2} R T_{i n t} .
\end{gathered}
$$

We define

$$
P=\rho R T_{e q} .
$$

As before, we also introduce the opposite of the stress tensor

$$
\rho \underline{\underline{\Theta}}=<<\underline{c} \otimes \underline{c} f>>
$$

still with $\underline{c}=\underline{v}-\underline{u}$. Next, we have to introduce two relaxation parameters $0 \leq \theta \leq 1$ and $-\frac{1}{2} \leq \nu<1$. We define a relaxation temperature

$$
T_{r e l}=\theta T_{e q}+(1-\theta) T_{i n t},
$$

a corrected tensor

$$
\underline{\underline{\mathcal{T}}}=(1-\theta)\left((1-\nu) R T_{t r} \underline{\underline{\underline{I d}}}+\nu \underline{\underline{\Theta}}\right)+\theta R T_{e q} \underline{\underline{I d}},
$$

and the generalized Gaussian

$$
\tilde{\mathcal{G}}[f]=\frac{\rho \Lambda_{\delta}}{\sqrt{\operatorname{det}(2 \pi \underline{\mathcal{T}})}\left(R T_{r e l}^{\delta / 2}\right)} \exp \left(-\frac{1}{2}(\underline{v}-\underline{u}) \cdot \underline{\underline{\mathcal{T}}}^{-1} \cdot(\underline{v}-\underline{u})+\frac{I^{2 / \delta}}{R T_{r e l}}\right)
$$

For later purpose, we also define the polyatomic maxwellian

$$
\tilde{\mathcal{M}}[f]=\Lambda_{\delta} \frac{\rho}{\left(2 \pi R T_{e q}\right)^{3 / 2}\left(R T_{e q}\right)^{\delta / 2}} \exp -\left(\frac{1}{2 R T_{e q}}|\underline{v}-\underline{u}|^{2}+\frac{I^{2 / \delta}}{R T_{e q}}\right) .
$$

We are now able to define the Gaussian-BGK polyatomic collision model

$$
Q(f)=\frac{P}{\mu(1-\nu+\theta \nu)}(\tilde{\mathcal{G}}[f]-f) .
$$

Here, the constant $\Lambda_{\delta}$ is defined by

$$
\Lambda_{\delta}^{-1}=\int e^{-I^{2} / \delta} d I
$$

so that

$$
<<\tilde{\mathcal{G}}[f]>>=<<f>>
$$


the collision operator (3.11) does preserve mass, momentum and energy, since we have

$$
\begin{gathered}
<<\underline{v} \tilde{\mathcal{G}}[f]>>=<<\underline{v} f>>, \\
<<\left(\frac{1}{2}|\underline{v}|^{2}+I^{2 / \delta}\right) \tilde{\mathcal{G}}[f]>>=<<\left(\frac{1}{2}|v|^{2}+I^{2 / \delta}\right) f>>.
\end{gathered}
$$

The first two properties are only due to the scalings of the constant $\Lambda_{\delta}$. The third conservation law can be checked as follows

$$
\begin{gathered}
<<\frac{|c|^{2}}{2} \tilde{\mathcal{G}}[f]>>=\frac{1}{2} \operatorname{tr} \underline{\underline{\mathcal{T}}}=\frac{3}{2} \rho\left(R T_{t r}(1-\theta)+\theta R T_{e q}\right), \\
<<I^{2 / \delta} \tilde{\mathcal{G}}[f]>>=\frac{\delta}{2} \rho R T_{r e l}=\frac{\delta}{2} \rho\left(\theta R T_{e q}+(1-\theta) R T_{i n t}\right),
\end{gathered}
$$

and the total internal energy is then

$$
<<\left(\frac{1}{2}|c|^{2}+I^{2 / \delta}\right) \tilde{\mathcal{G}}[f]>>=\rho R\left(\theta T_{e q}\left(\frac{\delta}{2}+\frac{3}{2}\right)+(1-\theta)\left(\frac{3}{2} T_{t r}+\frac{\delta}{2} T_{\text {int }}\right)\right)
$$

Since

$$
\frac{3}{2} T_{t r}+\frac{\delta}{2} T_{i n t}=\left(\frac{3}{2}+\frac{\delta}{2}\right) T_{e q}
$$

we obtain

$$
<<\left(\frac{1}{2}|\underline{v}|^{2}+I^{2 / \delta}\right) \tilde{\mathcal{G}}[f]>>=\frac{3+\delta}{2} \rho R T_{e q}=<<\left(\frac{1}{2}|v|^{2}+I^{2 / \delta}\right) f>>.
$$

We will now show that the modified BGK tensor (3.11) satisfies the entropy inequality. In section 4 we show that it gives, in the Chapman-Enskog expansion, the Prandtl number

$$
\frac{2}{3} \leq \operatorname{Pr}=\frac{1}{1-\nu+\theta \nu}<+\infty
$$

and a second viscosity coefficient in (1.2)

$$
\alpha=(\gamma-1)-\frac{1-\theta}{\theta}(1-\nu)\left(\frac{5}{3}-\gamma\right)
$$

always for $-\frac{1}{2} \leq \nu<1,0<\theta \leq 1$. 


\subsection{Entropy inequality}

To prove the entropy inequality (1.6) (H-Theorem) we introduce the entropy constrained minimization problem

$$
S\left(\rho, \underline{\underline{u}}, \underline{\underline{\mathcal{T}}}, T_{r e l}\right)=\min _{g \in \mathcal{X}}<<H(g)>>
$$

where $\mathcal{X}=\mathcal{X}\left(\rho, \underline{u}, \underline{\underline{\mathcal{T}}}, T_{\text {rel }}\right)$ is the set defined by

$$
\begin{aligned}
\mathcal{X} & =\left\{g \geq 0,\left(1+|v|^{2}\right) g \in L^{1}\left(I R^{3}\right),<<I^{2 / \delta} g>>=\frac{\delta}{2} R T_{r e l}\right. \\
& <<g>>=\rho,<<\underline{v} g>>=\rho \underline{u},<<\underline{v} \otimes \underline{v} g>>=\rho \underline{u} \otimes \underline{u}+\rho \underline{\mathcal{T}}\} .
\end{aligned}
$$

Our main result is the following proposition

\section{Proposition 3.1 .}

For symmetric positive definite tensor $\underline{\underline{\Theta}},-1 / 2 \leq \nu<1$, and $0<\theta<1$ we have

(i) the tensor $\underline{\underline{\mathcal{T}}}$ defined in (3.8) is symmetric positive definite and the set $\mathcal{X}\left(\rho, \underline{u}, \underline{\mathcal{T}}, T_{\text {rel }}\right)$ is not empty,

(ii) the unique minimizer is the Gaussian $\tilde{\mathcal{G}}[f]$ defined in (3.9),

(iii) the entropy of the Gaussian $\tilde{\mathcal{G}}[f]$ satisfies

$$
<<H(\tilde{\mathcal{G}})>>=S\left(\rho, \underline{u}, \underline{\underline{\mathcal{T}}}, T_{r e l}\right) \leq S\left(\rho, \underline{u}, \underline{\underline{\Theta}}, T_{i n t}\right) \leq<<H(f)>>,
$$

(iv) consequently the entropy inequalities (1.6), (1.7) hold,

(v) the equality $<<H(\tilde{\mathcal{G}})>>=<<H(f)>>$ implies $f=\tilde{\mathcal{M}}$.

Proof. From now on we note

$$
\underline{\underline{\mathcal{A}}}=\nu \underline{\underline{\Theta}}+(1-\nu) R T_{t r} \underline{\underline{I d}},
$$

and in case of $\theta=0$ we have $\underline{\underline{\mathcal{T}}}=\underline{\underline{\mathcal{A}}}$, especially this is true in the monoatomic case. To prove the first point, after noticing that $\tilde{\mathcal{G}}[f]$ belongs to $\mathcal{X}\left(\rho, \underline{u}, \underline{\mathcal{T}}, T_{r e l}\right)$ it is sufficient to prove that $\underline{\underline{\mathcal{A}}}$ is positive definite. Then, we can conclude the positivity of $\underline{\underline{\mathcal{T}}}$ by convexity since $0 \leq \theta<1$. To prove the positivity of $\underline{\underline{\mathcal{A}}}$, we consider a basis of eigenvectors of $\underline{\underline{\Theta}}$. In such a basis, the modified tensor $\underline{\underline{\mathcal{A}}}$ is diagonal, with diagonal terms

$$
A_{i}=\frac{(1-\nu)}{3}\left(\theta_{1}+\theta_{2}+\theta_{3}\right)+\nu \theta_{i}, \quad i=1,2 \text { or } 3 .
$$


These numbers are obviously positive when $0 \leq \nu \leq 1$. For negative values of $\nu$, the worst case occurs when two eigenvalues $\theta_{j}$ vanish, yielding for the other eigenvalue

$$
A_{i}=\frac{1+2 \nu}{3} \theta_{i} \geq 0, \quad \forall \nu \geq-\frac{1}{2},
$$

and the first point is proved.

To prove (ii), we first observe that from the definition of $\tilde{\mathcal{G}}[f]$, we have

$$
H^{\prime}(\tilde{\mathcal{G}})=\ln \tilde{\mathcal{G}}=\underline{\alpha} \cdot \underline{m}(\underline{v})
$$

where

$$
\underline{m}(\underline{v})={ }^{t}\left(1, \underline{v}, \underline{v} \otimes \underline{v}, I^{2 / \delta}\right),
$$

and with $\underline{\alpha}$ the Lagrange multiplier

$$
{ }^{t} \underline{\alpha}=\left(\ln \frac{\Lambda_{\delta} \rho}{\sqrt{\operatorname{det} 2 \pi \underline{\mathcal{T}}}\left(R T_{r e l}\right)^{\delta / 2}}-\frac{1}{2} u \cdot \underline{\underline{\mathcal{T}}}^{-1} u, \underline{\underline{\mathcal{T}}}^{-1} u,-\frac{1}{2} \underline{\underline{\mathcal{T}}}^{-1}, \frac{1}{R T_{r e l}}\right) .
$$

Since $H$ is strictly convex, we have, for all $g \geq 0$ with $g \neq \tilde{\mathcal{G}}$,

$$
H(g)>H(\tilde{\mathcal{G}})+H^{\prime}(\tilde{\mathcal{G}})(g-\tilde{\mathcal{G}}) .
$$

Thus, for $g \in \mathcal{X}\left(\rho, \underline{u}, \underline{\underline{T}}, T_{\text {rel }}\right)$ with $g \neq \tilde{\mathcal{G}}$, it follows from (3.15) and the definition of $\mathcal{X}\left(\rho, \underline{u}, \underline{\underline{\mathcal{T}}}, \bar{T}_{r e l}\right)$ that

$$
\int_{I R^{3}} H(g) d \underline{v}>\int_{I R^{3}} H(\tilde{\mathcal{G}}) d \underline{v} .
$$

This proves the second point.

For the third point, we first compute

$$
\begin{aligned}
& S\left(\rho, \underline{u}, \underline{\underline{\mathcal{T}}}, T_{r e l}\right)=\ln \left(\frac{\Lambda_{\delta} \rho}{\sqrt{\operatorname{det}(2 \pi \underline{\mathcal{T}})}\left(R T_{r e l}\right)^{\delta / 2}}\right)<<\tilde{\mathcal{G}}>>-\frac{1}{2}<<\underline{\underline{\mathcal{T}}}^{-1}:(\underline{c} \otimes \underline{c}) \tilde{\mathcal{G}}>> \\
& +<<\frac{I^{2 / \delta} \tilde{\mathcal{G}}}{R T_{\text {rel }}}>>-<<\tilde{\mathcal{G}}>> \\
& =\rho \ln \left(\frac{\Lambda_{\delta} \rho}{\sqrt{\operatorname{det}(2 \pi \underline{\mathcal{T}})}\left(R T_{r e l}\right)^{\delta / 2}}\right)-\frac{3}{2} \rho
\end{aligned}
$$

yielding

$$
\begin{aligned}
S\left(\rho, \underline{u}, \underline{\underline{\mathcal{T}}}, T_{r e l}\right)-S\left(\rho, \underline{u}, \underline{\underline{\Theta}}, T_{i n t}\right) & =\rho \ln \left(\frac{\Lambda_{\delta} \rho}{\sqrt{\operatorname{det}(2 \pi \underline{\mathcal{T}})}\left(R T_{r e l}\right)^{\delta / 2}}\right)-\rho \ln \left(\frac{\Lambda_{\delta} \rho}{\sqrt{\operatorname{det}(2 \pi \underline{\underline{\Theta}})}\left(R T_{\text {int }}\right)^{\delta / 2}}\right) \\
& =\frac{1}{2} \rho \ln \frac{\operatorname{det} \underline{\underline{\underline{\Theta}}} T_{r e l}^{\delta}}{\operatorname{det} \underline{\underline{\mathcal{T}}} T_{\text {int }}^{\delta}}
\end{aligned}
$$

RR $n^{\circ} 3716$ 
Thus, we just have to prove that

$$
\operatorname{det} \underline{\underline{\mathcal{T}}} T_{\text {rel }}^{\delta} \geq \operatorname{det} \underline{\underline{\Theta}} T_{\text {int }}^{\delta},
$$

We already proved in $(2.15)$ this inequality in the monoatomic case, i.e. $\underline{\underline{\mathcal{T}}}=\underline{\underline{\mathcal{A}}}$ and $\delta=0$

$$
\operatorname{det} \underline{\underline{\mathcal{A}}} \geq \operatorname{det} \underline{\underline{\Theta}} \text {. }
$$

Using this result for the polyatomic case, it is enough to prove the inequality

$$
\operatorname{det} \underline{\underline{\mathcal{T}}} T_{r e l}^{\delta} \geq \operatorname{det} \underline{\underline{\mathcal{A}}} T_{\text {int }}^{\delta} .
$$

First, we use again the Brunn-Minkowsky inequality (2.16) applied to $\underline{\underline{\mathcal{T}}}$

$$
\begin{array}{r}
\operatorname{det} \underline{\underline{\mathcal{T}}} \geq(\operatorname{det} \underline{\underline{\mathcal{A}}})^{1-\theta}\left(R T_{e q}\right)^{3 \theta} \\
\operatorname{det} \underline{\underline{\mathcal{T}}} \geq \operatorname{det} \underline{\underline{\mathcal{A}}}(\operatorname{det} \underline{\underline{\mathcal{A}}})^{-\theta}\left(R T_{e q}\right)^{3 \theta} .
\end{array}
$$

Then, we use the inequality between geometric and arithmetic average

$$
\left(\frac{\operatorname{tr} M}{d}\right)^{d} \geq \operatorname{det} M
$$

which gives here

$$
(\operatorname{det} \underline{\underline{\mathcal{A}}})^{-\theta} \geq\left(R T_{t r}\right)^{-3 \theta}
$$

since $-\theta \leq 0$ and $\operatorname{tr} \underline{\underline{\mathcal{A}}}=3 R T_{\text {tr }}$. We obtain thus

$$
\operatorname{det} \underline{\underline{\mathcal{T}}} \geq(\operatorname{det} \underline{\underline{\mathcal{A}}}) T_{e q}^{3 \theta} T_{t r}^{-3 \theta}
$$

Comparing with inequality 3.16 , it is sufficient to prove

$$
T_{e q}^{3 \theta} T_{t r}^{-3 \theta} T_{r e l}^{\delta} \geq T_{i n t}^{\delta},
$$

wich can be written

$$
3 \theta \ln T_{e q}-3 \theta \ln T_{t r}+\delta \ln T_{r e l} \geq \delta \ln T_{i n t} .
$$

For this we use the convex combination

$$
\begin{gathered}
T_{e q}=\frac{3}{3+\delta} T_{t r}+\frac{\delta}{3+\delta} T_{i n t} \\
T_{r e l}=(1-\theta) T_{i n t}+\frac{3 \theta}{3+\delta} T_{t r}+\frac{\delta \theta}{3+\delta} T_{i n t},
\end{gathered}
$$

and by the concavity of the logarithm fonction, it is a simple computation to obtain the inequality (3.18). 
This ends the demonstration of (iii).

We deduce (iv) as a consequence of (iii) and of the convexity relation

$$
H^{\prime}(f)(\tilde{\mathcal{G}}-f) \leq H(\tilde{\mathcal{G}})-H(f) .
$$

Then, the BGK model (3.11) satisfies the H-Theorem for all $\nu \in[-1 / 2,1], \theta \in$ $[0,1]$, because, using (iii)

$$
\begin{aligned}
\partial_{t} \int_{I R^{3}} H(f) d \underline{v}+\nabla_{\underline{x}} \cdot \int_{I R^{3}} \underline{v} H(f) d \underline{v} & =\frac{\rho R T}{\mu(1-\nu)} \int_{I R^{3}} H^{\prime}(f)(\tilde{\mathcal{G}}[f]-f) d \underline{v} \\
& \leq \frac{\rho R T}{\mu(1-\nu)} \int_{I R^{3}}(H(\tilde{\mathcal{G}}[f])-H(f)) d \underline{v} \\
& \leq 0 .
\end{aligned}
$$

The proof of $(\mathrm{v})$ is very much the same as in the monoatomic case. First, the equality $<<H(\tilde{\mathcal{G}})>>=<<H(f)>>$ implies

$$
S\left(\rho, \underline{u}, \underline{\underline{\Theta}}, T_{\text {int }}\right)=<<H(f)>>\text {, }
$$

and by (ii)

$$
f=\tilde{\mathcal{G}}_{\underline{\underline{\Theta}}, T_{i n t}}[f]=\frac{\rho \Lambda_{\delta}}{\sqrt{\operatorname{det}(2 \pi \underline{\underline{\Theta}})}\left(R T_{i n t}\right)^{\delta / 2}} \exp \left(-\frac{1}{2}(\underline{v}-\underline{u}) \cdot \underline{\underline{\Theta}}^{-1}(\underline{v}-\underline{u})+\frac{I^{2 / \delta}}{R T_{\text {int }}}\right) .
$$

Then, since $S\left(\rho, \underline{u}, \underline{\underline{\mathcal{T}}}, T_{\text {rel }}\right)=S\left(\rho, \underline{u}, \underline{\underline{\Theta}}, T_{\text {int }}\right)$, the calculation in the proof of (iii) gives

$$
\operatorname{det} \underline{\underline{\mathcal{T}}} T_{r e l}^{\delta}=\operatorname{det} \underline{\underline{\Theta}} T_{\text {int }}^{\delta}
$$

and also

$$
T_{e q}^{3(1-\theta)} T_{t r}^{3(\theta-1)} T_{r e l}^{\delta}=T_{i n t}^{\delta} .
$$

Next we take the logarithm of this equality, use the convex combinations which define $T_{r e l}$ and $T_{e q}$ and the strict concavity of the logarithm, and we deduce that the case of equality can only be reached by

$$
T_{\text {int }}=T_{t r}=T_{e q}
$$

Then, (3.20) gives

$$
\operatorname{det} \underline{\underline{\mathcal{T}}}=\operatorname{det} \underline{\underline{\Theta}}
$$

which has been proved in the monoatomic case to imply

$$
\underline{\underline{\Theta}}=R T_{t r}=R T_{e q} .
$$

This directly proves that

$$
f=\tilde{\mathcal{M}}[f] .
$$




\section{Transport coefficients}

We now compute the transport coefficients obtained from this modified BGK model in the Navier-Stokes approximation.

Proposition 4.1 For the Gaussian-BGK model, we obtain in the ChapmanEnskog expansion the Navier-Stokes system (1.2) where the viscosity tensor is given by

$$
\sigma_{i j}=\mu\left(\partial_{x_{j}} u_{i}+\partial_{x_{i}} u_{j}-\alpha \delta_{i j} \operatorname{div} u\right) .
$$

Moreover the second visosity coefficient $\alpha$ and Prandtl number $\frac{\gamma}{\gamma-1} \frac{R \mu}{\kappa}$ are given by

$$
\begin{gathered}
\alpha=(\gamma-1)-\frac{1-\theta}{\theta}(1-\nu)\left(\frac{5}{3}-\gamma\right), \\
\operatorname{Pr}=\frac{1}{1-\nu+\theta \nu} .
\end{gathered}
$$

The coefficients defined in $\S 3,-1 / 2 \leq \nu<1$ and $0<\theta<1$ lead to $\frac{2}{3} \leq \operatorname{Pr}<+\infty$ in formula (4.3). For a monoatomic gaz, a Prandlt of $2 / 3$ is obtained by taking $\theta=0$ and $\nu=-\frac{1}{2}$. For a diatomic gaz, experimental values of the Prandtl are close to $\frac{5}{7}$ and this is obtained with $\nu=-\frac{1}{2}$ and $\theta=\frac{1}{5}$. This value of $\theta$ corresponds to the empirical law that in a diatomic gas, one collision of particles out of five involves an exchange of internal energy, as is widely used in Monte Carlo (DSMC) simulations.

\section{Proof.}

The proof is based on a Chapman-Enskog expansion of the unknown distribution $f$, verifying

$$
\partial_{t} f+\underline{v} \cdot \nabla_{\underline{x}} f=\frac{P}{\mu(1-\nu)}(\tilde{\mathcal{G}}[f]-f) \text {. }
$$

We denote

$$
\epsilon=\frac{\mu(1-\nu+\theta \nu)}{P}
$$

and thus write

$$
f=\tilde{\mathcal{G}}[f]-\epsilon\left(\partial_{t} f+\underline{v} \cdot \nabla_{x} f\right) .
$$

We then expand $f$ as a power serie of $\epsilon$. We obtain immediatly from the above equation that the first term of the expansion -taking formally $\epsilon=0$ - verifies $f^{0}=\tilde{\mathcal{G}}\left[f^{0}\right]$ wich implies, using proposition $3.1(\mathrm{v})$ that

$$
f^{0}=\tilde{\mathcal{M}}\left[f^{0}\right]
$$

In a second step, we consider the first correction in $\epsilon$. We therefore write $f^{\epsilon}=\tilde{\mathcal{M}}\left[f^{\epsilon}\right]+O(\epsilon)$. Inserting this in (4.5) we obtain

$$
f^{\epsilon}=\tilde{\mathcal{G}}\left[f^{\epsilon}\right]-\epsilon\left(\partial_{t} \mathcal{M}\left[f^{\epsilon}\right]+\underline{v} \cdot \nabla_{x} \mathcal{M}\left[f^{\epsilon}\right]\right)+0\left(\epsilon^{2}\right) .
$$


On the other hand, integrating the kinetic equation in mass, momentum and energy, and recalling the definition of $\rho, u, T_{e q}$ in (3.1)-(3.2)-(3.3), we have the conservation laws

$$
\partial_{t}\left(\begin{array}{l}
\rho \\
\rho u_{i} \\
E
\end{array}\right)+\partial_{x_{j}} \cdot\left(\begin{array}{l}
\rho u_{j} \\
\rho u_{i} u_{j}+\rho \Theta_{i j}^{\epsilon} \\
E u_{j}+\rho \Theta_{i j}^{\epsilon} u_{i}+q_{j}^{\epsilon}
\end{array}\right)=0
$$

under the notation

$$
\begin{gathered}
<<\left(\frac{1}{2}|c|^{2}+I^{2 / \delta}\right) c_{j} f^{\epsilon}>>=q_{j}{ }^{\epsilon}, \\
<<v_{i} v_{j} f^{\epsilon}>>=\rho u_{i} u_{j}+\rho \Theta_{i j}^{\epsilon} .
\end{gathered}
$$

Therefore, to obtain the comportment law (4.1) and the Fourier law, we only need to compute the moments $<<v_{i} v_{j} f^{\epsilon}>>$ and $<<v_{i} v_{j} v_{k} f^{\epsilon}>>$. We compute them up to the first order of $\epsilon$, using (4.6) and the following lemma

\section{Lemma 4.2}

$$
\begin{aligned}
\partial_{t}<<v_{i} v_{j} \tilde{\mathcal{M}} & >+\partial_{x_{k}}<<v_{i} v_{j} v_{k} \tilde{\mathcal{M}}>>=P\left(\frac{\partial u_{i}}{\partial x_{j}}+\frac{\partial u_{j}}{\partial x_{i}}-(\gamma-1) \frac{\partial u_{k}}{\partial x_{k}} \delta_{i j}\right) \\
\partial_{t} & <<\left(\frac{1}{2} v^{2}+I^{2 / \delta}\right) v_{j} \tilde{\mathcal{M}}>>+\partial_{x_{k}}<<\left(\frac{1}{2} v^{2}+I^{2 / \delta}\right) v_{j} v_{k} \tilde{\mathcal{M}}>> \\
& =P u_{i}\left[\frac{\partial u_{i}}{\partial x_{j}}+\frac{\partial u_{j}}{\partial x_{i}}-(\gamma-1) \frac{\partial u_{k}}{\partial x_{k}} \delta_{i j}\right]+\frac{5+\delta}{2} P R \frac{\partial T}{\partial x_{j}} .
\end{aligned}
$$

This lemma is mainly deduced from standard algebraic manipulations and exact computation of the third and fourth moment of the maxwellian, and we do not recall the computations.

Reporting this in (4.6), and using the definitions of the first moments of the modified tensor $\mathcal{G}\left(f^{\epsilon}\right)$, we therefore get

$$
\begin{aligned}
<<f^{\epsilon} v_{i} v_{j}>>= & \rho u_{i} u_{j}+\theta \rho R T_{e q} \delta_{i j}+(1-\theta) \rho\left((1-\nu) R T_{t r}^{\epsilon} \delta_{i j}+\nu \Theta_{i j}^{\epsilon}\right) \\
& -\epsilon P\left(\partial_{x_{j}} u_{i}+\partial_{x_{i}} u_{j}-(\gamma-1) \partial_{x_{k}} u_{k} \delta_{i j}\right)+0\left(\epsilon^{2}\right) \\
<<\left(\frac{1}{2}|c|^{2}+I^{2 / \delta}\right) c_{j} f^{\epsilon}>>= & \left(\frac{1}{2} \rho u^{2}+\frac{3+\delta}{2} \rho R T_{e q}^{\epsilon}\right) u_{j}+\theta \rho R T_{e q}^{\epsilon} \delta_{i j} u_{i} \\
& +(1-\theta) \rho\left((1-\nu) R T_{t r}^{\epsilon} \delta_{i j}+\nu \Theta_{i j}^{\epsilon}\right) u_{i} \\
& -\epsilon P\left(\partial_{x_{j}} u_{i}+\partial_{x_{i}} u_{j}-(\gamma-1) \partial_{x_{k}} u_{k} \delta_{i j}\right) u_{i}-\epsilon \frac{5+\delta}{2} R P \partial_{x_{j}} T+0\left(\epsilon^{2}\right) .
\end{aligned}
$$

RR $n^{\circ} 3716$ 
Using the above definitions (4.8) and (4.9) we obtain

$$
\begin{aligned}
\rho \Theta_{i j}^{\epsilon}= & \theta \rho R T_{e q} \delta_{i j}+(1-\theta) \rho\left((1-\nu) R T_{t r}^{\epsilon} \delta_{i j}+\nu \Theta_{i j}^{\epsilon}\right) \\
& -\epsilon P\left(\partial_{x_{j}} u_{i}+\partial_{x_{i}} u_{j}-(\gamma-1) \partial_{x_{k}} u_{k} \delta_{i j}\right)+0\left(\epsilon^{2}\right), \\
q_{j}^{\epsilon}+\rho \Theta_{i j}^{\epsilon} u_{i}= & \theta \rho R T_{e q}^{\epsilon} \delta_{i j} u_{i}+(1-\theta) \rho\left((1-\nu) R T_{t r}^{\epsilon} \delta_{i j}+\nu \Theta_{i j}^{\epsilon}\right) u_{i} \\
& -\epsilon P\left(\partial_{x_{j}} u_{i}+\partial_{x_{i}} u_{j}-(\gamma-1) \partial_{x_{k}} u_{k} \delta_{i j}\right) u_{i}-\epsilon \frac{5+\delta}{2} R P \partial_{x_{j}} T+0\left(\epsilon^{2}\right) .
\end{aligned}
$$

Multiplying the first equations by $u_{i}$ and substracting them to the second ones gives then

$$
q_{j}^{\epsilon}=-\epsilon \frac{5+\delta}{2} R P \partial_{x_{j}} T+0\left(\epsilon^{2}\right) .
$$

On the other hand, taking the trace of the first equations first gives

$$
3 \rho R T_{t r}^{\epsilon}=3 \theta \rho R T_{e q}^{\epsilon}+3(1-\theta) \rho R T_{t r}^{\epsilon}-\epsilon P(5-3 \gamma) \partial_{x_{k}} u_{k},
$$

that is

$$
R T_{t r}^{\epsilon}=R T_{e q}^{\epsilon}-\frac{1}{\theta} \epsilon P\left(\frac{5}{3}-\gamma\right) \partial_{x_{k}} u_{k},
$$

and therefore the first equations reduce finally to

$(1-\nu+\theta \nu) \rho \Theta_{i j}^{\epsilon}=(1-\nu+\theta \nu) \rho R T_{e q}^{\epsilon} \delta_{i j}-\epsilon P\left(\partial_{x_{j}} u_{i}+\partial_{x_{i}} u_{j}-\alpha \partial_{x_{k}} u_{k} \delta_{i j}\right)+O\left(\epsilon^{2}\right)$,

with $\alpha=(\gamma-1)-\frac{1-\theta}{\theta}(1-\nu)\left(\frac{5}{3}-\gamma\right)$.

Substituting $\epsilon$ by its value $\epsilon=\frac{\mu(1-\nu+\theta \nu)}{P}$ gives the final result

$$
\begin{array}{r}
\rho \Theta_{i j}^{\epsilon}=P \delta_{i j}-\mu\left(\partial_{x_{j}} u_{i}+\partial_{x_{i}} u_{j}-\alpha \partial_{x_{k}} u_{k} \delta_{i j}\right)+O\left(\epsilon^{2}\right), \\
q_{j}^{\epsilon}=-\frac{5+\delta}{2} \mu(1-\nu+\theta \nu) R \partial_{x_{j}} T+0\left(\epsilon^{2}\right)=-\kappa \partial_{x_{j}} T+0\left(\epsilon^{2}\right) .
\end{array}
$$

At first order in $\epsilon$, these are the right Navier-Stokes constitutive laws with Prandtl number $\operatorname{Pr}=\frac{\gamma}{\gamma-1} \frac{R \mu}{\kappa}=\frac{5+\delta}{2} \frac{R \mu}{\kappa}=\frac{1}{1-\nu+\nu \theta}$.

\section{Polyatomic case. Description with two distri- bution functions}

For numerical purposes, it is expensive to introduce an internal energy variable. It turns out that, for BGK models that is not necessary and all macroscopic 
variables can be equivalently obtained with a simpler formalism. We simply need to introduce two density distributions, one for mass and one for internal energy. Notice that the reduction below cannot be performed in the case of Boltzmann's quadratic collision operator.

Using the notations of $\S 3$, we denote $\tilde{f}(t, x, v)$ the solution of the Boltzmann equation and we define

$$
\begin{aligned}
& f(t, \underline{x}, \underline{v})=\int_{I R^{+}} \tilde{f}(t, \underline{x}, \underline{v}, I) d I, \\
& g(t, \underline{x}, \underline{v})=\int_{I R^{+}} I^{2 / \delta} \tilde{\tilde{f}}(t, \underline{x}, \underline{v}, I) d I .
\end{aligned}
$$

The macroscopic quantities of $\S 3$ are then simply defined in function of $f$ and $g$ by

$$
\begin{aligned}
& \rho=\left\langle f>, \rho u=\left\langle\underline{v} f>, \underline{\underline{\Theta}}=\frac{1}{\rho}<\underline{c} \otimes \underline{c} f>\right.\right. \\
& e=\frac{3+\delta}{2} R T_{e q}=\frac{3}{2} R T_{t r}+\frac{\delta}{2} R T_{i n t}, \\
& \frac{3 R}{2} T_{t r}=\operatorname{Tr} \underline{\underline{\Theta}}, \quad e_{i n t}=\frac{\delta R}{2} T_{i n t}=\frac{1}{\rho}<g>.
\end{aligned}
$$

This allows to construct the associated Gaussian distribution

$$
\begin{aligned}
& \underline{\underline{\mathcal{T}}}=(1-\nu) R T_{t r} \underline{\underline{I d}}+\nu \underline{\underline{\Theta}}, \\
& \mathcal{G}[f, g]=\int_{I R_{+}} \tilde{\mathcal{G}}[\tilde{f}] d I \\
& =\frac{\rho}{\sqrt{\operatorname{det}(2 \pi \underline{\mathcal{T}})}} \exp \left(-\frac{1}{2}(\underline{v}-\underline{u}) \cdot \underline{\underline{T}}^{-1} \cdot(\underline{v}-\underline{u})\right) \\
& \mathcal{M}[f, g]=\int_{I R_{+}} \tilde{\mathcal{M}}[\tilde{f}] d I \\
& =\frac{\rho}{\left(2 \pi T_{e q}\right)^{\frac{3}{2}}} \exp \left(-\frac{\underline{\underline{v}}-\left.\underline{u}\right|^{2}}{2 T_{e q}}\right) \text {. }
\end{aligned}
$$

Then, we obtain the BGK system governing the evolution of the distributions $f$ and $g$ by simply integrating the kinetic equation (1.1), (3.11) in $d I$ and $I^{2 / \delta} d I$, yielding

$$
\begin{aligned}
& \partial_{t} f+v \cdot \nabla_{x} f+\frac{1}{\varepsilon}(f-\mathcal{G}[f, g])=0, \\
& \partial_{t} g+v \cdot \nabla_{x} g+\frac{1}{\varepsilon}\left(g-\frac{\delta}{2} R T_{r e l} \mathcal{G}[f, g]\right)=0 .
\end{aligned}
$$

For this system the only conservation quantities are again

$$
\rho, \rho \underline{u}, E=\frac{1}{2} \rho|\underline{u}|^{2}+\frac{3}{2} \rho R T_{t r}+\frac{\delta \rho}{2} R T_{\text {int }} .
$$

RR $n^{\circ} 3716$ 
Since the macroscopic quantities associated with this system are the same as those of $f$ in $\S 3$, we also obtain, in the Chapman-Enskog expansion the same Prandtl number

$$
\frac{2}{3} \leq \operatorname{Pr}=\frac{1}{1-\nu+\theta \nu}<+\infty .
$$

Finally, the entropy property now holds in average for the H-function defined by

$$
\begin{aligned}
& H(f, g)=f \ln \frac{f}{(g)^{\frac{\delta}{2+\delta}}}-f \\
& \quad=\operatorname{Min}\left\{\int_{I R_{+}} H(\tilde{f}) d I ; f \geq 0, \int_{I R_{+}} \tilde{f} d I=f, \int_{I R_{+}} I^{2 / \delta} \tilde{f} d I=g\right\} .
\end{aligned}
$$

Indeed, we can prove as in Propositions 2.1 - 3.1 that the pair $\left(\mathcal{M}, \frac{\delta}{2} R T_{e q} \mathcal{M}\right)$ minimizes $<H(f, g)>$ under the constraints

$$
<f>=\rho,<\underline{v} f>=\rho \underline{u}, E=<\frac{v^{2}}{2} f+g>,
$$

while the pair $\left(\mathcal{G}, \frac{\delta}{2} R T_{\text {rel }} \mathcal{G}\right)$ minimizes $<H(f, g)>$ under the constraints

$$
\begin{aligned}
& <f>=\rho,<\underline{v} f>=\rho \underline{u},\langle\underline{c} \otimes \underline{c} f>=\rho \underline{\underline{\mathcal{T}}} \\
& <g>=\rho \frac{\delta}{2} R T_{\text {rel }} .
\end{aligned}
$$

This implies in turn

$$
<H\left(\mathcal{G}, \frac{\delta}{2} R T_{r e l} \mathcal{G}\right)>=S\left(\rho, \underline{u}, \underline{\underline{\mathcal{T}}}, T_{r e l}\right) \leq S\left(\rho, \underline{u}, \underline{\underline{\Theta}}, T_{i n t}\right) \leq<H(f, g)>
$$

which proves that

$$
\begin{aligned}
\partial_{t} & <H(f, g)>+\operatorname{div}<\underline{v} H(f, g)> \\
& \leq \frac{1}{\varepsilon}<H\left(\mathcal{G}, \frac{\delta}{2} R T_{r e l} \mathcal{G}\right)-H(f, g)> \\
& \leq 0 .
\end{aligned}
$$

\section{Appendix. Brunn-Minkowsky inequality.}

We give here a simple demonstration of the Brunn-Minkowsky inequality introduced in section 2 and 3 .

$$
\operatorname{det}(a A+(1-a) B)>(\operatorname{det} A)^{a}(\operatorname{det} B)^{1-a}
$$


for $0<a<1$ and $A$ positive, $B$ nonnegative symmetric matrices, $A \neq B$.

To prove this inequality, we can chose $A=\mathrm{Id}$ without any loss of generality. This is consequence of the strict positivity of one of the two matrices, for example $A$, so that $A$ is invertible, and

$$
\operatorname{det}(a A+(1-a) B)=\operatorname{det}(A) \operatorname{det}\left(a \mathrm{Id}+(1-a) A^{-1} B\right) .
$$

Changing the the matrices names, we have only to prove

$$
\operatorname{det}(a \operatorname{Id}+(1-a) C)>(\operatorname{det} C)^{1-a} .
$$

Here $C=A^{-1} B$ is still diagonalisable and we denote by $c_{i}$ its eigenvalues. Computing the determinants in a diagonal basis for $C$,

$$
\Pi\left(a+(1-a) c_{i}\right)>\left(\Pi c_{i}\right)^{1-a} .
$$

Taking the logarithm, we have to prove that

$$
\Sigma \ln \left(a+(1-a) c_{i}\right)>(1-a) \Sigma \ln c_{i} .
$$

This is now a direct consequence of the concavity of the logarithm between 1 and $c_{i}$

$$
\ln \left(a+(1-a) c_{i}\right)>(1-a) \ln c_{i}
$$

\section{References}

[1] P. Andries, J.F. Bourgat, B. Perthame, and P. Le Tallec. Comparison between the Boltzmann and ES-BGK model by a Monte Carlo simulation. In preparation.

[2] K. Aoki, K. Kanba, and S. Takata. Numerical analysis of a supersonic rarefied gas flow past a flat plate. Phys. Fluids, 9(4):1144-1161, 1997.

[3] P.L. Bhatnagar, E.P. Gross, and M. Krook. A model for collision processes in gases. Phys. Rev., 94:511, 1954.

[4] G.-A. Bird. Molecular Gas Dynamics and the Direct Simulation of Gas Flow. Oxford Engineering Science Series Publications, 1994.

[5] F. Bouchut and B. Perthame. A BGK model for small Prandtl number in the Navier-Stokes approximation. Journal Stat. Physics, 71(1-2):191-207, 1993.

RR $n^{\circ} 3716$ 
P. Andries, P. Le Tallec, J.P. Perlat, B.Perthame

[6] R. Brun. Transport et relaxation dans les écoulements gazeux. Masson, 1986 .

[7] C. Cercignani. Phys. Fluids, 1859, 1967.

[8] C. Cercignani. The Boltzmann Equation and its Applications. Applied Mathematical Sciences, Springer-Verlag., 1975.

[9] L. H. Holway. Kinetic theory of shock structure using an ellipsoidal distribution function. In Rarefied Gas Dynamics, Vol. I (Proc. Fourth Internat. Sympos., Univ. Toronto, 1964), pages 193-215. Academic Press, New York, 1966.

[10] M.S. Ivanov and S.V. Rogasinsky. Analysis of numerical techniques of direct simulation Monte-Carlo method in the rarefied gas dynamics. $J$. Numer. Anal. Math. Modelling, 3(6):453-465, 1988.

[11] B. Khobalatte and B. Perthame. Maximum principle on the entropy and minimal limitations for kinetic schemes. Math. Comp., 17(205):119-131, 1994.

[12] C. Levermore. Moment closure hierarchies for kinetic theories. J. Statist. Phys., 83(5-6):1021-1065, 1996.

[13] E.M. Lifschitz and L.P. Pitaevski. Physical kinetics. Pergamon Press, Oxford, 1981.

[14] S. K. Loyalka, N. Petrellis, and T. S. Storvick. Some exact numerical results for the BGK model: Couette, Poiseuille and thermal creep flow between parallel plates. Z. Angew. Math. Phys., 30(3):514-521, 1979.

[15] L. Mieussens. Discrete velocity model and implicite scheme for the BGK equation of rarefied gas dynamics. Math. Models and Meth. Appl. Sci., preprint.

[16] T.F. Morse. A kinetic model for gases with internal degrees of freedom. Phys. Fluids, 7:159-169, 1964.

[17] W. G. Vincenti and C. H. Kruger. Introduction to Physical Gas Dynamics. Robert E. Krieger Publishing Company., 1965. 
Unit ${ }^{\prime}$ de recherche INRIA Lorraine, Technopôle de Nancy-Brabois, Campus scientifique, 615 rue du Jardin Botanique, BP 101, 54600 VILLERS LÈS NANCY

Unit ${ }^{\prime} e$ de recherche INRIA Rennes, Irisa, Campus universitaire de Beaulieu, 35042 RENNES Cedex

Unit'e de recherche INRIA Rhône-Alpes, 655, avenue de l'Europe, 38330 MONTBONNOT ST MARTIN

Unit'e de recherche INRIA Rocquencourt, Domaine de Voluceau, Rocquencourt, BP 105, 78153 LE CHESNAY Cedex

Unit'e de recherche INRIA Sophia-Antipolis, 2004 route des Lucioles, BP 93, 06902 SOPHIA-ANTIPOLIS Cedex

Éditeur

INRIA, Domaine de Voluceau, Rocquencourt, BP 105, 78153 LE CHESNAY Cedex (France)

http://www.inria.fr

ISSN 0249-6399 